\title{
Existence and stability of solutions for a strongly coupled system modelling thin fluid films
}

\author{
Joachim Escher and Bogdan-Vasile Matioc
}

\begin{abstract}
In this paper we consider a strongly coupled fourth order parabolic system modelling the motion of two thin fluid layers in the presence of gravity and surface tension. In the non-degenerate case we prove existence and uniqueness of strong solutions and study the stability of the equilibria for various fluid-fluid configurations.
\end{abstract}

Mathematics Subject Classification. 76A20, 35Q35, 35B35, 35K52.

Keywords. Thin film, Strong solutions, Stability, Strongly coupled, Degenerate system.

\section{Introduction}

In this paper we study a parabolic system modeling two-phase flows in porous media which are driven by capillary and gravity forces

$$
\left\{\begin{array}{l}
\partial_{t} f=\partial_{x}\left[f\left(-A \partial_{x}^{3} f-B \partial_{x}^{3} h+a \partial_{x} f+b \partial_{x} h\right)\right], \\
\partial_{t} h=\partial_{x}\left[h\left(-\partial_{x}^{3} f-\partial_{x}^{3} h+c \partial_{x} f+c \partial_{x} h\right)\right],
\end{array} \quad \text { for }(t, x) \in(0, \infty) \times(0, L),\right.
$$

whereby $L \in(0, \infty)$. The system (1.1a) is supplemented by initial conditions for $f$ and $h$ of the form

$$
f(0)=f_{0}, \quad h(0)=h_{0},
$$

and no-flux boundary conditions at $x=0$ and $x=L$

$$
\partial_{x} f=\partial_{x} h=\partial_{x}^{3} f=\partial_{x}^{3} h=0, \quad \text { at } \quad x=0, L .
$$

Furthermore, the constants involved in (1.1a) are assumed to satisfy

$$
a, b, c \in[0, \infty), \quad c B=b, \quad \text { and } \quad A>B>0 .
$$


The model has been obtained in [6] by passing to the limit of thin fluid threads in the full two-phase Muskat problem considered in [7]. It is worth noticing that similar methods have been used in [9] and [14] to find, in the limit of thin fluid threads, thin film equations as approximations for the capillary drive Stokes and Hele-Shaw moving boundary value problems. Compared to the Muskat problem which can be expressed as a nonlocal and nonlinear evolution equation, (1.3) is a local, quasilinear system which does not have the complexity of the original problem. In order to give a physical interpretation to system (1.1a), we recall the system obtained in (1.3)

$$
\left\{\begin{array}{c}
\partial_{t} f=\partial_{x}\left[-\mu_{-}^{-1} \widetilde{\gamma} f \partial_{x}^{3} \tilde{f}-\mu_{-}^{-1} \gamma f \partial_{x}^{3} f+\mu_{-}^{-1} g\left(\rho_{-}-\rho_{+}\right) f \partial_{x} f+\mu_{-}^{-1} g \rho_{+} f \partial_{x} \tilde{f}\right] \\
\partial_{t} \widetilde{f}=\partial_{x}\left[-\mu_{+}^{-1} \widetilde{\gamma}(\widetilde{f}-f) \partial_{x}^{3} \widetilde{f}-\mu_{-}^{-1} \widetilde{\gamma} f \partial_{x}^{3} \widetilde{f}-\mu_{-}^{-1} \gamma f \partial_{x}^{3} f+\mu_{+}^{-1} g \rho_{+}(\widetilde{f}-f) \partial_{x} \widetilde{f}\right. \\
\left.\quad+\mu_{-}^{-1} g\left(\rho_{-}-\rho_{+}\right) f \partial_{x} f+\mu_{-}^{-1} g \rho_{+} f \partial_{x} \tilde{f}\right]
\end{array}\right.
$$

for $(t, x) \in(0, \infty) \times(0, L)$. Therein, the line $y=0$ is the bottom (assumed to be impermeable) of the porous medium. The region between the graph $y=f(t, x) \geq 0$ and the bottom $y=0$ is occupied by a fluid having density $\rho_{-}$ and viscosity $\mu_{-}$, and above the graph $y=f(t, x)$ we have a fluid with density $\rho_{+}$and viscosity $\mu_{+}$. This second liquid is separated by the air (assumed at constant pressure) by an interface parametrised by the function $\widetilde{f}(t, x)$. The non-degenerate case, which we consider in this paper, corresponds to the situation when $\tilde{f}>f>0$. As usual, $g$ stands for the gravity constant, $\gamma$ is the surface tension coefficient at the interface $y=f(t, x)$, while $\widetilde{\gamma}$ is the surface tension coefficient at the interface $y=\widetilde{f}(t, x)$.

Mathematically, it is more useful to re-express the system in the variables $(f, h)$, where $h:=\widetilde{f}-f$ is a measure for the thickness of the layer located above. By doing this, and defining

$$
a:=\frac{g \rho_{-}}{\widetilde{\gamma}} \frac{\mu_{+}}{\mu_{-}}, \quad b:=\frac{g \rho_{+}}{\widetilde{\gamma}} \frac{\mu_{+}}{\mu_{-}}, \quad c:=\frac{g \rho_{+}}{\widetilde{\gamma}}, \quad A:=\frac{\gamma+\widetilde{\gamma}}{\widetilde{\gamma}} \frac{\mu_{+}}{\mu_{-}}, \quad B:=\frac{\mu_{+}}{\mu_{-}},
$$

we arrive, after rescaling the time in (1.3) according to $\widetilde{t}:=\mu_{+}^{-1} \widetilde{\gamma} t$ (we drop the tilde thereafter), at system (1.1a). We note that when one of the functions $f$ or $h$ is constantly equal to zero, the other unknown is a solution of the thin film equation with nonlinear diffusion whose investigation has and still receives a lot of attention $[3,10,15-17,20]$. In the absence of surface tension effects, that is when $A=B=0$, problem (1.1) has been analyzed in [6] for strong solutions, and in [4] in the degenerate case when the two interfaces may enter in contact or touch the bottom. Nonnegative weak solutions in the pure capillary case when $a=b=c=0$ have been constructed in [13] and, as those in [6], they are shown to exist globally.

In this paper we are interested in the existence of strong solutions for the system (1.1) when there exists a competition between capillary and gravity forces. By studying the linearization of the equations, it turns out that 
we deal with a parabolic problem. For the second order system analyzed in [6] well established parabolic theory is available, cf. [2]. In contrast, in the present paper we derive the needed a priori estimates by introducing weighted scalar products which dependent on the point where we linearize and by using the Lax-Milgram theorem. Compared to the full Muskat problem [7], where the linearization is a $2 \times 2$-matrix generator whose off-diagonal entries are of lower order, herein we obtain a matrix with elements having all the same order. Additionally, we identify the steady-state solutions of the problem and study their stability properties. The steady states are either flat, or when the fluid below is less dense then that above, there may exist non-constant steady-state solutions of (1.1). They form one-dimensional linear center manifolds which may be regarded as the first order expansions of the analytic bifurcation branches consisting of non-flat steady-state solutions of the Muskat problem in [7].

The outline of the paper is as follows: in Sect. 2 we express the problem as an abstract quasilinear evolution equation and derive the estimates which are needed for our local well-posedness result (see Theorem 2.1). In Sect. 3 we study the stability properties of the steady-state solutions by using the principle of linearized stability and, in the critical case of stability, a center manifold analysis, cf. Theorems 3.3 and 3.6.

\section{The quasilinear evolution equation}

We start our analysis by introducing the spaces we work with. Throughout the paper, we set $L_{2}:=L_{2}\left((0, L), \mathbb{R}^{2}\right), H^{k}:=H^{k}\left((0, L), \mathbb{R}^{2}\right)$, and, given $k \in \mathbb{N}$,

$$
\begin{gathered}
H_{\mathcal{B}}^{k}:=\left\{X:=(f, h) \in H^{k}: \partial_{x}^{2 l+1} X(0)=\partial_{x}^{2 l+1} X(L)=0\right. \\
\text { for all } l \in \mathbb{N} \text { with } 2 l+2 \leq k\} .
\end{gathered}
$$

These spaces are endowed with the usual Sobolev norms. An important role in our analysis is played by the space $H_{\mathcal{B}}^{4}$. We also need the corresponding complex interpolation spaces with $L_{2}$, that is $\left[L_{2}, H_{\mathcal{B}}^{4}\right]_{\theta}=: H_{\mathcal{B}}^{4 \theta}, \theta \in[0,1] \backslash\{3 / 8,7 / 8\}$. It follows from Theorem 4.3.3 in [21] that

$$
H_{\mathcal{B}}^{4 \theta}:= \begin{cases}H^{4 \theta}\left((0, L), \mathbb{R}^{2}\right), & 0 \leq \theta \leq 3 / 8 \\ \left\{X \in H^{4 \theta}\left((0, L), \mathbb{R}^{2}\right): \partial_{x} X=0 \text { for } x=0, L\right\}, & 3 / 8<\theta \leq 7 / 8 \\ \left\{X \in H^{4 \theta}\left((0, L), \mathbb{R}^{2}\right): \partial_{x} X=\partial_{x}^{3} X=0 \text { for } x=0, L\right\}, 7 / 8<\theta \leq 1 .\end{cases}
$$

In the classical setting considered in this paper we do not allow that the interfaces touch or meet the bottom of the medium. Therefore, we define

$$
\mathcal{O}:=\left\{X=(f, h) \in H_{\mathcal{B}}^{3}: f>0 \text { and } h>0\right\},
$$

which is an open subset of $H_{\mathcal{B}}^{3}$, and, for each $X \in \mathcal{O}$, we introduce the matrix

$$
a(X):=\left[\begin{array}{cc}
A f & B f \\
h & h
\end{array}\right]
$$

and the mapping $\mathcal{A}: \mathcal{O} \rightarrow \mathcal{L}\left(H_{\mathcal{B}}^{4}, L_{2}\right)$ by the relation

$$
\mathcal{A}(X) Y:=\partial_{x}\left(a(X) \partial_{x}^{3} Y\right), \quad X \in \mathcal{O}, Y \in H_{\mathcal{B}}^{4} .
$$


Note that $\mathcal{A}(X)$ is a linear operator for each $X \in \mathcal{O}$. Furthermore, the lower order terms in (1.1a) are comprised by the function $f: \mathcal{O} \rightarrow L_{2}$, given by

$$
f(X):=\left(\begin{array}{l}
\partial_{x}\left[f\left(a \partial_{x} f+b \partial_{x} h\right)\right] \\
\partial_{x}\left[h\left(c \partial_{x} f+c \partial_{x} h\right)\right]
\end{array}\right) .
$$

With this notation, our problem (1.1) may be re-expressed as a quasilinear evolution equation:

$$
\partial_{t} X+\mathcal{A}(X) X=f(X), \quad t>0, \quad X(0)=X_{0},
$$

and we find ourselves in the abstract setting presented in Section 12 of [2]. Our first main result is the following well-posedness statement.

Theorem 2.1. Let $\alpha>3 / 4$. Given $\left(f_{0}, h_{0}\right) \in \mathcal{O} \cap H_{\mathcal{B}}^{4 \alpha}$, the problem (1.1) possesses a unique maximal strong solution

$(f, h) \in C\left([0, T), \mathcal{O} \cap H_{\mathcal{B}}^{4 \alpha}\right) \cap C^{\alpha}\left([0, T), L_{2}\right) \cap C^{1}\left((0, T), L_{2}\right) \cap C\left((0, T), H_{\mathcal{B}}^{4}\right)$,

with the maximal existence time $T \in(0, \infty]$.

Proof. This result is a direct consequence of Theorems 2.2 and 2.3 below.

We recall now an abstract result [2, Theorem 12.1] which is one of the main ingredients in the proof of Theorem 2.1.

Theorem 2.2. (Theorem 12.1 in [2]) Let $\left(E_{0}, E_{1}\right)$ be a densely injected Banach couple and $(\cdot, \cdot)_{\theta}$ an admissible interpolation functor. Given $\mathcal{O} \subset E_{\theta}:=$ $\left(E_{0}, E_{1}\right)_{\theta}$ for some $\theta \in(0,1)$, let $\mathcal{O}_{\eta}:=\mathcal{O} \cap E_{\eta}$ for $\eta \in[\theta, 1]$.

Suppose that $0<\gamma \leq \beta<\alpha<1$, that $\mathcal{O}_{\beta}$ is open in $E_{\beta}$, and that

$$
(\mathcal{A}, f) \in C^{1-}\left(\mathcal{O}_{\beta}, \mathcal{H}\left(E_{1}, E_{0}\right) \times E_{\gamma}\right) .
$$

Then, the autonomous quasilinear parabolic Cauchy problem (2.2) possesses for each $X \in \mathcal{O}_{\alpha}$ a unique maximal solution

$$
X \in C\left([0, T), \mathcal{O}_{\alpha}\right) \cap C^{\alpha}\left([0, T), E_{0}\right) \cap C^{1}\left((0, T), E_{0}\right) \cap C\left((0, T), E_{1}\right) .
$$

In order to apply Theorem 2.2 to our particular situation, we identify $E_{0}:=L_{2}, E_{1}:=H_{\mathcal{B}}^{4}$, and $(\cdot, \cdot)_{\theta}$ to be the complex interpolation functor $[\cdot, \cdot]_{\theta}$. Furthermore, we let $\gamma=1 / 4$ and $\beta=3 / 4$, meaning that $E_{\gamma}=H_{\mathcal{B}}^{1}$ and $E_{\beta}=H_{\mathcal{B}}^{3}$. It is clear from the definition of $\mathcal{A}$ and $f$ that

$$
(\mathcal{A}, f) \in C^{\infty}\left(\mathcal{O}, \mathcal{L}\left(H_{\mathcal{B}}^{4}, L_{2}\right) \times H_{\mathcal{B}}^{1}\right),
$$

where $\mathcal{O}$ is the open subset of $H_{\mathcal{B}}^{3}$ given by (2.1). In the reminder of this section we prove that the operator $\mathcal{A}(X), X \in \mathcal{O}$, is, as an unbounded operator in $L_{2}$ with definition domain $H_{\mathcal{B}}^{4}$, the negative generator of an analytic semigroup in $\mathcal{L}\left(L_{2}\right)$.

Theorem 2.3. Given $\bar{X}=(\bar{f}, \bar{h}) \in \mathcal{O}$, the operator $-\mathcal{A}(\bar{X})$ generates a strongly continuous and analytic semigroup in $\mathcal{L}\left(L_{2}\right)$, that is

$$
\mathcal{A}(\bar{X}) \in \mathcal{H}\left(H_{\mathcal{B}}^{4}, L_{2}\right) .
$$


In order to prove Theorem 2.4, we pick $\bar{X}=(\bar{f}, \bar{h}) \in \mathcal{O}$ and define the unbounded operator $\mathbb{A}:=-\mathcal{A}(\bar{X}): H_{\mathcal{B}}^{4} \subset L_{2} \rightarrow L_{2}$ by

$$
\mathbb{A}(X):=\left(\begin{array}{c}
-\partial_{x}\left[\bar{f} \partial_{x}^{3}(A f+B h)\right] \\
-\partial_{x}\left[\bar{h} \partial_{x}^{3}(f+h)\right]
\end{array}\right), \quad \forall X=(f, h) \in H_{\mathcal{B}}^{4} .
$$

Furthermore, using $\bar{X}=(\bar{f}, \bar{h})$, we define a weighted scalar product on $H_{\mathcal{B}}^{k}$, $k \in \mathbb{N}$, by the relation

$$
(X \mid \widetilde{X})_{k}:=\sum_{i=0}^{k} \int_{0}^{L} \bar{h} \partial_{x}^{i} f \partial_{x}^{i} \tilde{f}+B \bar{f} \partial_{x}^{i} h \partial_{x}^{i} \widetilde{h} d x
$$

for $X=(f, h)$ and $\widetilde{X}=(\tilde{f}, \widetilde{h})$ in $H_{\mathcal{B}}^{k}, B$ being the constant given by (1.2). Since $\bar{f}$ and $\bar{h}$ are both positive and continuous functions, we easily see that the norm $\|\cdot\|_{k}$ induced by this scalar product is equivalent to the usual Sobolev norm $\|\cdot\|_{H^{k}}$ on $H_{\mathcal{B}}^{k}, k \in \mathbb{N}$, meaning that $\left(H_{\mathcal{B}}^{k},(\cdot \mid \cdot)_{k}\right)$ is a Hilbert space for all $k \in \mathbb{N}$. By doing this, we encapsulate some of the structure of the system (1.1a) into the spaces we work with.

Lemma 2.4. There exist positive constants $c, C$ such that

$$
\|\mathbb{A} X\|_{0} \geq c\|X\|_{4}-C\|X\|_{0} \quad \text { for all } X \in H_{\mathcal{B}}^{4} \text {. }
$$

Proof. Let $X=(f, h) \in H_{\mathcal{B}}^{4}$ be given. We then have:

$$
\begin{aligned}
\|\mathbb{A} X\|_{0}^{2} & =\int_{0}^{L} \bar{h}\left\{\partial_{x}\left[\bar{f} \partial_{x}^{3}(A f+B h)\right]\right\}^{2}+B \bar{f}\left\{\partial_{x}\left[\bar{h} \partial_{x}^{3}(f+h)\right]\right\}^{2} d x \\
& \geq m \int_{0}^{L}\left\{\partial_{x}\left[\bar{f} \partial_{x}^{3}(A f+B h)\right]\right\}^{2}+\left\{\partial_{x}\left[\bar{h} \partial_{x}^{3}(f+h)\right]\right\}^{2} d x
\end{aligned}
$$

where $m:=\min \{\bar{f}, \bar{h}, B \bar{f}\}$. Taking into account that $(x+y)^{2} \geq 3 x^{2} / 4-4 y^{2}$ for all $x, y \in \mathbb{R}$, we conclude

$$
\begin{aligned}
\|\mathbb{A} X\|_{0}^{2} \geq & \frac{3 m}{4} \int_{0}^{L} \bar{f}^{2}\left[\partial_{x}^{4}(A f+B h)\right]^{2}+\bar{h}^{2}\left[\partial_{x}^{4}(f+h)\right]^{2} d x \\
& -3 m \int_{0}^{L}\left(\partial_{x} \bar{f}\right)^{2}\left[\partial_{x}^{3}(A f+B h)\right]^{2}+\left(\partial_{x} \bar{h}\right)^{2}\left[\partial_{x}^{3}(f+h)\right]^{2} d x .
\end{aligned}
$$

Since by (1.2) we have $A>B$, we may use Poincaré's inequality and the fact that $\bar{X} \in H_{\mathcal{B}}^{3}$, to finally arrive at $(2.6)$.

It follows easily from Lemma 2.4 that $\mathbb{A}$ is a closed operator. Note further that the structure of the weighted Sobolev norms has not been used in an essential way in the proof of Lemma 2.4. We shall take now benefit of the particular structure of (2.5) and establish coercivity estimates in higher Sobolev spaces.

Lemma 2.5. (Coercivity estimates) There exist positive constants $c, C$ such that

$$
(-\mathbb{A} X \mid X)_{2 k} \geq c\|X\|_{2(k+1)}^{2}-C\|X\|_{2 k}^{2}
$$

for all $X \in H_{\mathcal{B}}^{2(k+2)}, k \in\{0,1\}$. 
Proof. We observe that the assumption $\bar{X} \in H_{\mathcal{B}}^{3}$ ensures that $\mathbb{A}: H_{\mathcal{B}}^{6} \rightarrow H_{\mathcal{B}}^{2}$ is a well-defined operator. Let us investigate the more involved case $k=1$ first. Given $X=(f, g) \in H_{\mathcal{B}}^{6}$, the highest order term in the definition of $(-\mathbb{A} X \mid X)_{2}$ is

$$
\begin{aligned}
(- & \left.\partial_{x}^{2} \mathbb{A} X \mid \partial_{x}^{2} X\right)_{0}=\int_{0}^{L} \bar{h} \partial_{x}^{2} f \partial_{x}^{3}\left[\bar{f} \partial_{x}^{3}(A f+B h)\right]+B \bar{f} \partial_{x}^{2} h \partial_{x}^{3}\left[\bar{h} \partial_{x}^{3}(f+h)\right] d x \\
= & \int_{0}^{L} \partial_{x}^{2}\left(\bar{h} \partial_{x}^{2} f\right) \partial_{x}\left[\bar{f} \partial_{x}^{3}(A f+B h)\right]+B \partial_{x}^{2}\left(\bar{f} \partial_{x}^{2} h\right) \partial_{x}\left[\bar{h} \partial_{x}^{3}(f+h)\right] d x \\
= & \int_{0}^{L} \bar{f} \bar{h} \partial_{x}^{4} f \partial_{x}^{4}(A f+B h)+B \bar{f} \bar{h} \partial_{x}^{4} h \partial_{x}^{4}(f+h) d x \\
& +\int_{0}^{L}\left(\partial_{x}^{2} \bar{h} \partial_{x}^{2} f+2 \partial_{x} \bar{h} \partial_{x}^{3} f\right) \partial_{x}\left[\bar{f} \partial_{x}^{3}(A f+B h)\right]+\partial_{x} \bar{f} \partial_{x}^{2}\left(\bar{h} \partial_{x}^{2} f\right) \partial_{x}^{3}(A f+B h) d x \\
& +B \int_{0}^{L}\left(\partial_{x}^{2} \bar{f} \partial_{x}^{2} h+2 \partial_{x} \bar{f} \partial_{x}^{3} h\right) \partial_{x}\left[\bar{h} \partial_{x}^{3}(f+h)\right]+\partial_{x} \bar{h} \partial_{x}^{2}\left(\bar{f} \partial_{x}^{2} h\right) \partial_{x}^{3}(f+h) d x \\
\geq & \left(\frac{(A-B) m^{2}}{2}\left\|\partial_{x}^{4} f\right\|_{L_{2}((0, L), \mathbb{R})}^{2}+\frac{m^{2} B(A-B)}{4(A+B)}\left\|\partial_{x}^{4} h\right\|_{\left.L_{2}((0, L), \mathbb{R})\right)}^{2}\right) \\
& -C\|X\|_{4}\|X\|_{3},
\end{aligned}
$$

with $m$ as in the proof of Lemma 2.4. Using Young's together with Poincaré's inequality, we find that $\left(-\partial_{x}^{2} \mathbb{A} X \mid \partial_{x}^{2} X\right)_{0}$ is bounded from below by the expression on the right-hand side of relation (2.7). Proceeding similarly, we obtain that

$$
\left(-\partial_{x} \mathbb{A} X \mid \partial_{x} X\right)_{0} \geq c\|X\|_{3}^{2}-C\|X\|_{1}^{2} \quad \text { and } \quad(-\mathbb{A} X \mid X)_{0} \geq c\|X\|_{2}^{2}-C\|X\|_{0}^{2},
$$

which completes our proof.

Relations (2.6) and (2.7) are used later to show that if $\omega \in \mathbb{R}$ is sufficiently large, then the operator $(\mathbb{A}-\omega)$ is one-to-one and has closed range. ${ }^{1}$ In order to show that, for such $\omega,(\mathbb{A}-\omega)$ is also onto, we use the operator

$$
\left(1-\partial_{x}^{2}\right):\left\{f \in H^{2}((0, L), \mathbb{R}): \partial_{x} f=0 \text { for } x=0, L\right\} \rightarrow L_{2}((0, L), \mathbb{R}),
$$

which is well-known to be an isomorphism, cf. [4], and construct a bilinear form closely related to the operator $\mathbb{A}$. We extended herein some ideas which appear in [19] in the context of the Stokes and Hele-Shaw moving boundary value problems. The bilinear form $\mathcal{B}: H_{\mathcal{B}}^{4} \times H_{\mathcal{B}}^{4} \rightarrow \mathbb{R}$ is defined by the relation

$$
\begin{aligned}
\mathcal{B}(X, \tilde{X}):= & \sum_{i=0}^{2} \int_{0}^{L} \partial_{x}^{i}\left(1-\partial_{x}^{2}\right)^{-1}\left\{\partial_{x}\left[\bar{f} \partial_{x}^{3}(A f+B h)\right]\right\}\left(1-\partial_{x}^{2}\right)\left(\bar{h} \partial_{x}^{i} \widetilde{f}\right) d x \\
& +B \sum_{i=0}^{2} \int_{0}^{L} \partial_{x}^{i}\left(1-\partial_{x}^{2}\right)^{-1}\left\{\partial_{x}\left[\bar{h} \partial_{x}^{3}(f+h)\right]\right\}\left(1-\partial_{x}^{2}\right)\left(\bar{f} \partial_{x}^{i} \widetilde{h}\right) d x
\end{aligned}
$$

\footnotetext{
${ }^{1}$ Observe that the principal part of $\mathcal{A}(\bar{X})$ corresponds to a strongly coupled system which is in addition non-symmetric. Therefore, the determination of the Fredholm index is not obvious.
} 
for all $X=(f, h)$ and $\tilde{X}=(\tilde{f}, \tilde{h})$ in $H_{\mathcal{B}}^{4}$. By the definition, it is clear that $\mathcal{B}$ is continuous. Furthermore, we observe that, if in addition $X \in H_{\mathcal{B}}^{6}$, then we have

$$
\mathcal{B}(X, \tilde{X})=(-\mathbb{A} X \mid \widetilde{X})_{2}
$$

Relation (2.8) is a consequence of the fact that $\partial_{x}^{i}\left(1-\partial_{x}^{2}\right)^{-1} f=\left(1-\partial_{x}^{2}\right)^{-1} \partial_{x}^{i} f$ for all $f \in H^{2}((0, L), \mathbb{R})$ which satisfy homogeneous Neumann conditions at $x=0, L$, and of the identity

$$
\int_{0}^{L} h\left(1-\partial_{x}^{2}\right)^{-1} f d x=\int_{0}^{L} f\left(1-\partial_{x}^{2}\right)^{-1} h d x, \quad f, h \in L_{2}((0, L), \mathbb{R}) .
$$

Choose now $\omega \in \mathbb{R}$ sufficiently large, to guarantee that the bilinear form $\mathcal{B}_{\lambda}: H_{\mathcal{B}}^{4} \times H_{\mathcal{B}}^{4} \rightarrow \mathbb{R}$

$$
\mathcal{B}_{\lambda}(X, \tilde{X}):=\lambda(X \mid \widetilde{X})_{2}+\mathcal{B}(X, \tilde{X}), \quad X, \tilde{X} \in \mathbb{E}_{4}
$$

is coercive for all $\lambda \geq \omega$. This is possible, since by (2.8) and (2.7) (with $k=1$ ) we have

$$
\mathcal{B}_{\lambda}(X, X) \geq \lambda\|X\|_{2}^{2}+c\|X\|_{4}^{2}-C\|X\|_{2}^{2} \geq c\|X\|_{4}^{2}
$$

for all $X \in H_{\mathcal{B}}^{6}$ and $\lambda \geq \omega \geq C$. Using the density of $H_{\mathcal{B}}^{6}$ in $H_{\mathcal{B}}^{4}$ and the continuity of $\mathcal{B}_{\lambda}$ we obtain that $(2.9)$ is satisfied by all elements $X \in H_{\mathcal{B}}^{4}$. The Lax-Milgram theorem guarantees now the existence of an isomorphism $T_{\lambda}: H_{\mathcal{B}}^{4} \rightarrow H_{\mathcal{B}}^{4}$ with the property that

$$
\mathcal{B}_{\lambda}(X, \tilde{X})=\left(T_{\lambda} X \mid \widetilde{X}\right)_{4} \quad \text { for all } X, \widetilde{X} \in H_{\mathcal{B}}^{4}
$$

Particularly, by (2.8), it holds for $\lambda \geq \omega$ that

$$
-((\mathbb{A}-\lambda) X \mid \widetilde{X})_{2}=\left(T_{\lambda} X \mid \widetilde{X}\right)_{4} \quad \text { whenever } X \in H_{\mathcal{B}}^{6}
$$

Proof of Theorem 2.3. Gathering (2.6) and (2.7), we may choose the constant $\omega$ large enough to ensure, besides $(2.9)$, that $(\mathbb{A}-\lambda)$ is bounded from below, that is

$$
\|(\mathbb{A}-\lambda) X\|_{0} \geq C\|X\|_{4}
$$

for all $X \in H_{\mathcal{B}}^{4}$ and $\lambda \geq \omega$, with a positive constant $C$ which depends only on $\omega$. For $\lambda \geq \omega$, we let $T_{\lambda}$ denote the isomorphism given by (2.10). By (2.11), the operator $(\mathbb{A}-\lambda)$ is one-to-one and its range is closed if $\lambda \geq \omega$. Even more, its range is dense in $L_{2}$. Indeed, since $(\mathbb{A}-\lambda) \in \mathcal{L}\left(H_{\mathcal{B}}^{6}, H_{\mathcal{B}}^{2}\right)$, we let $M$ be the complement of the closure of $(\mathbb{A}-\lambda)\left(H_{\mathcal{B}}^{6}\right)$ in $H_{\mathcal{B}}^{2}$, that is $\overline{(\mathbb{A}-\lambda)\left(H_{\mathcal{B}}^{6}\right)} \oplus M=H_{\mathcal{B}}^{2}$. Then, we necessarily have $M \cap H_{\mathcal{B}}^{4}=\{0\}$. To prove this relation, pick $\tilde{X} \in H_{\mathcal{B}}^{4}$ and assume that $((\mathbb{A}-\lambda) X \mid \widetilde{X})_{2}=0$ for all $X \in H_{\mathcal{B}}^{6}$. Then, we obtain from (2.10) that $\widetilde{X}$ is orthogonal on the closure $\overline{T_{\lambda} H_{\mathcal{B}}^{6}}$ of $T_{\lambda} H_{\mathcal{B}}^{6}$ in $H_{\mathcal{B}}^{4}$. Since $H_{\mathcal{B}}^{6}$ is densely embedded in $H_{\mathcal{B}}^{4}$ and $T_{\lambda}$ is an isomorphism, it follows that $\overline{T_{\lambda} H_{\mathcal{B}}^{6}}=H_{\mathcal{B}}^{4}$, and therefore $\tilde{X}=0$. Whence $H_{\mathcal{B}}^{4} \subset \overline{(\mathbb{A}-\lambda)\left(H_{\mathcal{B}}^{6}\right)}$, and we have $\overline{(\mathbb{A}-\lambda)\left(H_{\mathcal{B}}^{6}\right)}=H_{\mathcal{B}}^{2}$. Lastly, we may use the density of $H_{\mathcal{B}}^{2}$ in $L_{2}$ to conclude that $(\mathbb{A}-\lambda)\left(H_{\mathcal{B}}^{6}\right)$ is dense in $L_{2}$. 
Summarising $\mathbb{A}-\lambda: H_{\mathcal{B}}^{4} \rightarrow L_{2}$ is an isomorphism for all $\lambda \geq \omega$, meaning that the non-negative half-axis is contained in the resolvent set of $\mathbb{A}-\omega$. Due to $(2.7)$ (with $k=0$ ), we obtain, since $\omega \geq C$, that additionally

$$
((\mathbb{A}-\omega) X \mid X)_{0} \leq-c\|X\|_{2}^{2}, \quad \forall X \in H_{\mathcal{B}}^{4} .
$$

Given $k \in \mathbb{N}$, we let $H_{\mathcal{B}, c}^{k}:=\left\{X_{r}+i X_{c}: X_{r}, X_{c} \in H_{\mathcal{B}}^{k}\right\}$ denote the complexification of $H_{\mathcal{B}}^{k}$, which is a Hilbert space with the scalar product

$$
\left(X_{r}+i X_{c} \mid \widetilde{X}_{r}+i \widetilde{X}_{c}\right)_{k, c}:=\left(X_{r} \mid \widetilde{X}_{r}\right)_{k}+\left(X_{c} \mid \widetilde{X}_{c}\right)_{k}-i\left(X_{r} \mid \widetilde{X}_{c}\right)_{k}+i\left(X_{c} \mid \widetilde{X}_{r}\right)_{k}
$$

For $k=0$, we write $L_{2, c}=: H_{\mathcal{B}, c}^{0}$. The complexification of $\mathbb{A}$ is the operator $\mathbb{A}^{c}: H_{\mathcal{B}, c}^{4} \rightarrow L_{2, c}$ with

$$
\mathbb{A}^{c}\left(X_{r}+i X_{c}\right):=\mathbb{A} X_{r}+i \mathbb{A} X_{c} \quad \text { for } X=X_{r}+i X_{c} \in H_{\mathcal{B}, c}^{4} .
$$

We infer now from (2.12) that for $X=X_{r}+i X_{c} \in H_{\mathcal{B}, c}^{4}$ we have

$$
\begin{aligned}
\operatorname{Re}\left(\left(\mathbb{A}^{c}-\omega\right) X \mid X\right)_{0, c} & =\left((\mathbb{A}-\omega) X_{r} \mid X_{r}\right)_{0}+\left((\mathbb{A}-\omega) X_{c} \mid X_{c}\right)_{0} \\
& \leq-c\left(\left\|X_{r}\right\|_{2}^{2}+\left\|X_{c}\right\|_{2}^{2}\right) \leq 0 \\
\left|\operatorname{Im}\left(\left(\mathbb{A}^{c}-\omega\right) X \mid X\right)_{0, c}\right| & \leq\left|\left((\mathbb{A}-\omega) X_{r} \mid X_{c}\right)_{0}\right|+\left|\left((\mathbb{A}-\omega) X_{c} \mid X_{r}\right)_{0}\right| \\
& \leq C\left(\left\|X_{r}\right\|_{2}\left\|X_{c}\right\|_{2}\right) \leq C\left(\left\|X_{r}\right\|_{2}^{2}+\left\|X_{c}\right\|_{2}^{2}\right) \\
& \leq-(C / c) \operatorname{Re}((\mathbb{A}-\omega) X \mid X)_{0, c},
\end{aligned}
$$

where we used integration by parts in order to obtain the second inequality when estimating the imaginary part of $\left(\left(\mathbb{A}^{c}-\omega\right) X \mid X\right)_{0}$. By virtue of (2.13), we conclude that the numerical range of the operator $\mathbb{A}^{c}-\omega$, i.e.

$$
S\left(\mathbb{A}^{c}-\omega\right):=\left\{\left(\left(\mathbb{A}^{c}-\omega\right) X \mid X\right): X \in H_{\mathcal{B}, c}^{4}\right\},
$$

is contained in a sector in the left halfplane which has angle less than $\pi$. Since $[0, \infty)$ belongs to the resolvent set of $\mathbb{A}^{c}-\omega$, we infer from Theorem 3.9 in [18] that the spectrum of $\mathbb{A}^{c}-\omega$ is contained in the same sector $\{z \in \mathbb{C}$ : $\operatorname{Re} z \leq 0$ and $|\operatorname{Im} z| \leq-(C / c) \operatorname{Re} z\}$ and

$$
\left\|\left(\left(\mathbb{A}^{c}-\omega\right)-\lambda\right)^{-1}\right\|_{\mathcal{L}\left(L_{2}^{c}\right)} \leq \frac{1}{d\left(\lambda, S\left(\mathbb{A}^{c}-\omega\right)\right)} \leq \frac{1}{|\lambda|} \quad \text { for all } \operatorname{Re} \lambda \geq \omega .
$$

Consequently, we obtain from Proposition 2.1.11 in [12] that $\mathbb{A}^{c}-\omega$ is sectorial, and together with Corollary 2.1.3 in [12] we find $\omega-\mathbb{A} \in \mathcal{H}\left(H_{\mathcal{B}}^{4}, L_{2}\right)$. This implies $-\mathbb{A} \in \mathcal{H}\left(H_{\mathcal{B}}^{4}, L_{2}\right)$, and the proof is complete.

\section{Steady-state solutions and stability properties}

Given $X:=(f, h) \in \mathcal{O}$, we introduce the energy functional

$$
\mathcal{E}(X)=: \mathcal{E}(f, h):=\int_{\mathbb{R}} \Phi(f)+B \Phi(h) d x,
$$

whereby $\Phi:[0, \infty) \rightarrow \mathbb{R}$ is the convex, non-negative function $\Phi(x):=x \ln (x)-$ $x+1, x \geq 0$. The lemma below shows that the energy of the solutions of (1.1) dissipates in time when the less dense fluid lies above, that is when $a>b$. We observe that, when considering (1.3), it is not clear at all that the functional $\mathcal{E}$ 
can be used to derive useful a priori estimates for that system. This emphasizes once more the importance of expressing (1.3) in the new variables $(f, h)$.

Lemma 3.1. Assume that $X=(f, h)$ is a solution of (1.1), as stated in Theorem 2.1. We then have:

$$
\begin{aligned}
& \frac{d}{d t} \mathcal{E}(X)+(A-B)\left\|\partial_{x}^{2} f\right\|_{L_{2}((0, L), \mathbb{R})}^{2}+B \|\left.\partial_{x}^{2}(f+h)\right|_{L_{2}((0, L), \mathbb{R})} ^{2} \\
& \quad+(a-b)\left\|\partial_{x} f\right\|_{L_{2}((0, L), \mathbb{R})}^{2}+b\left\|\partial_{x}(f+h)\right\|_{L_{2}((0, L), \mathbb{R})}^{2}=0,
\end{aligned}
$$

for all $t \in(0, T)$.

Proof. Let us first recall that, by $(1.2), c B=b$. Therefore, given $t \in(0, T)$, we find

$$
\begin{aligned}
\frac{d}{d t} \mathcal{E}(X)= & \int_{0}^{L} \Phi^{\prime}(f) \partial_{t} f+B \Phi^{\prime}(h) \partial_{t} h d x \\
= & \int_{0}^{L}\left\{\Phi^{\prime}(f) \partial_{x}\left[f\left(-A \partial_{x}^{3} f-B \partial_{x}^{3} h+a \partial_{x} f+b \partial_{x} h\right)\right]\right. \\
& \left.+B \Phi^{\prime}(h) \partial_{x}\left[h\left(-\partial_{x}^{3} f-\partial_{x}^{3} h+c \partial_{x} f+c \partial_{x} h\right)\right]\right\} d x \\
= & -\int_{0}^{L}\left\{\partial_{x} f\left(A \partial_{x}^{3} f+B \partial_{x}^{3} h-a \partial_{x} f-b \partial_{x} h\right)\right. \\
& \left.+B \partial_{x} h\left(\partial_{x}^{3} f+\partial_{x}^{3} h-c \partial_{x} f-c \partial_{x} h\right)\right\} d x \\
= & -\int_{0}^{L}\left\{A\left(\partial_{x}^{2} f\right)^{2}+2 B \partial_{x}^{2} f \partial_{x}^{2} h+B\left(\partial_{x}^{2} h\right)^{2}+a\left(\partial_{x} f\right)^{2}+2 b \partial_{x} f \partial_{x} h\right. \\
& \left.+b\left(\partial_{x} h\right)^{2}\right\} d x \\
= & -\int_{0}^{L}\left\{(A-B)\left(\partial_{x}^{2} f\right)^{2}+B\left(\partial_{x}^{2} f+\partial_{x}^{2} h\right)^{2}+(a-b)\left(\partial_{x} f\right)^{2}\right. \\
& \left.+b\left(\partial_{x} f+\partial_{x} h\right)^{2}\right\} d x,
\end{aligned}
$$

which is the desired relation (3.1).

As a direct consequence of Lemma 3.1 we identify, when the more dense fluid lies beneath or when difference of the fluids' densities is relatively small compared to the surface tension coefficient at the interface between them, the steady-state solutions of the problem.

Corollary 3.2. Assume that

$$
\frac{\pi^{2}}{L^{2}}(A-B)+(a-b)>0 .
$$

Then, the only stationary solutions of problem (1.1) are constant pairs $X_{*}=$ $\left(f_{*}, h_{*}\right)$, with $f_{*}, h_{*} \in(0, \infty)$. Moreover, if $(3.2)$ is not satisfied, then $X_{*}$ is still a stationary solution of (1.1).

Proof. Recall by Wirtinger's inequality that $\left\|\partial_{x} f\right\|_{L_{2}((0, L), \mathbb{R})} \leq(L / \pi)$ $\left\|\partial_{x}^{2} f\right\|_{L_{2}((0, L), \mathbb{R})}$ for all $f \in H^{2}((0, L), \mathbb{R})$ with $\partial_{x} f=0$ at $x=0, L$. Whence, if $X=(f, h)$ is a stationary solution of (1.1), i.e. $f$ and $h$ are independent of 
time, we obtain from (3.1) and (3.2) that $\partial_{x}^{2} f=\partial_{x}^{2} h=0$. Since $X \in H_{\mathcal{B}}^{4} \cap \mathcal{O}$, we conclude that both $f$ and $h$ must be positive constants.

When studying the stability properties of these equilibria, we have to take into account the property of the solutions of conserving the fluid volumes. Indeed, it follows directly from (1.1a) and (1.1c) that

$$
\frac{d}{d t} \int_{0}^{L} f(t, x) d x=\frac{d}{d t} \int_{0}^{L} h(t, x) d x=0
$$

as long as the solution $(f, h)$ exists. Our first stability result is the following theorem.

Theorem 3.3. (Stability properties of flat equilibria) Let $X_{*}=\left(f_{*}, h_{*}\right)$ be a steady-state solution of (1.1). If (3.2) is satisfied, then there exist positive constants $M, \omega, \delta$ such that for all $X_{0}=\left(f_{0}, h_{0}\right) \in H_{\mathcal{B}}^{4} \cap \mathcal{O}$ with

$$
\left\|X_{0}-X_{*}\right\|_{H^{4}} \leq \delta \quad \text { and } \quad \int_{0}^{L}\left(f_{0}-f_{*}, h_{0}-h_{*}\right) d x=(0,0),
$$

the solution $X=(f, h)$ of (1.1) exists globally and

$$
\left\|X(t)-X_{*}\right\|_{H^{4}}+\left\|\partial_{t} X(t)\right\|_{L_{2}} \leq M e^{-\omega t}\left\|X_{0}-X_{*}\right\|_{H^{4}}
$$

for all $t \in[0, \infty)$.

Moreover, if

$$
\frac{\pi^{2}}{L^{2}}(A-B)+(a-b)<0,
$$

then $X_{*}=\left(f_{*}, h_{*}\right)$ is unstable.

Remark 3.4. We notice that the flat equilibria are stable also when the more dense fluid lies above, i.e. $a<b$, provided the density jump across the interface is small compared to $A-B$. In the case when $L=\pi$ relation (3.2) is equivalent to $\gamma+g\left(\rho_{-}-\rho_{+}\right)>0$, which is exactly the condition found for the full Muskat problem studied in [7].

The critical case of stability when neither (3.2) nor (3.5) are satisfied will be discussed in Theorem 3.6.

In order to prove Theorem 3.3, we choose a steady-state $X_{*}$ and, as in $[5,6]$, we introduce the condition (3.3) into the spaces we work with. Let $P \in \mathcal{L}\left(L_{2}\right) \cap \mathcal{L}\left(H_{\mathcal{B}}^{4}\right)$ denote the orthogonal projection corresponding to the two-dimensional subspace spanned by constants

$$
P(X)=P(f, h)=\left(\frac{1}{L} \int_{0}^{L} f(x) d x, \frac{1}{L} \int_{0}^{L} h(x) d x\right) .
$$

If $X_{0}$ satisfies (3.4), then, by virtue of (3.3), the solution $X$ of (1.1) which corresponds to the initial data $X_{0}$ is given by

$$
X(t)=X_{*}+Y(t),
$$


with $Y(t):=(I-P) X(t) \in(I-P) H_{\mathcal{B}}^{4}$. This relation is an immediate consequence of the fact that we may decompose $H_{\mathcal{B}}^{4}$ and $L_{2}$ as direct sums:

$$
H_{\mathcal{B}}^{4}:=P H_{\mathcal{B}}^{4} \oplus(I-P) H_{\mathcal{B}}^{4} \quad \text { and } \quad L_{2}:=P L_{2} \oplus(I-P) L_{2} .
$$

Moreover, the function $Y(t)$ solves the problem

$$
\partial_{t} Y=F(Y):=-\mathcal{A}\left(X_{*}+Y\right) Y+f\left(X_{*}+Y\right), \quad t>0, \quad Y(0):=X_{0}-X_{*} .
$$

This means that the stability properties of the solution $X_{*}$ of (1.1) are determined by those of the solution $Y=0$ of (3.9). Let us first observe, by the definition of $\mathcal{A}$ and $f$, that the operator $F \in C^{\infty}\left(\mathcal{O}_{Y},(I-P) L_{2}\right), \mathcal{O}_{Y}:=$ $(I-P)\left(\mathcal{O} \cap H_{\mathcal{B}}^{4}\right)$ being a zero neighbourhood in $(I-P) H_{\mathcal{B}}^{4}$.

The proof of Theorem 3.3 is a direct consequence of the following lemma and of the principle of linearized stability, cf. [12, Theorem 9.1.2].

Lemma 3.5. Consider $\partial F(0)$ as an unbounded operator in $(I-P) L^{2}$ with domain of definition $(I-P) H_{\mathcal{B}}^{4}$. We then have:

(a) $\quad-\partial F(0) \in \mathcal{H}\left((I-P) H_{\mathcal{B}}^{4},(I-P) L^{2}\right)$;

(b) The spectrum $\sigma(\partial F(0))$ contains only the real eigenvalues

$$
\lambda_{1 / 2}^{k}:=r_{k}^{2} \frac{-\left[f_{*}\left(A r_{k}^{2}+a\right)+h_{*}\left(r_{k}^{2}+c\right)\right] \pm \sqrt{D_{k}}}{2},
$$

where $D_{k}:=\left[f_{*}\left(A r_{k}^{2}+a\right)+h_{*}\left(r_{k}^{2}+c\right)\right]^{2}-4 f_{*} h_{*}\left(r_{k}^{2}+c\right)[(a-b)+$ $\left.(A-B) r_{k}^{2}\right]$ and $r_{k}=k \pi / L$.

Proof. To prove (a), we note that since $X_{*}$ is constant in the space variable, the Fréchet derivative $\partial F(0)$ is given by $\partial F(0) Y=-\mathcal{A}\left(X_{*}\right) Y+\partial f\left(X_{*}\right) Y$ for all $Y \in(I-P) H_{\mathcal{B}}^{4}$. Therefore, we may write, with respect to the decompositions (3.8), the operator $\mathcal{A}\left(X_{*}\right)-\partial f\left(X_{*}\right) \in \mathcal{L}\left(H_{\mathcal{B}}^{4}, L_{2}\right)$ in matrix form, as follows

$$
\mathcal{A}\left(X_{*}\right)-\partial f\left(X_{*}\right)=\left[\begin{array}{ll}
-\partial F(0) & 0 \\
0 & 0
\end{array}\right] \in \mathcal{L}\left(H_{\mathcal{B}}^{4}, L_{2}\right) .
$$

Taking into account that

$$
\partial f\left(X_{*}\right)=\left[\begin{array}{ll}
a f_{*} & b f_{*} \\
c h_{*} & c h_{*}
\end{array}\right] \partial_{x}^{2} \in \mathcal{L}\left(H_{\mathcal{B}}^{2}, L_{2}\right),
$$

and $H_{\mathcal{B}}^{2}:=\left[H_{\mathcal{B}}^{4}, L_{2}\right]_{1 / 2}$ we may consider $\partial f\left(X_{*}\right)$ as a lower order perturbation of $-\mathcal{A}\left(X_{*}\right)$. We infer then from Theorem 2.3 and Proposition 2.4.1 in [12] that $\mathcal{A}\left(X_{*}\right)-\partial f\left(X_{*}\right) \in \mathcal{H}\left(H_{\mathcal{B}}^{4}, L_{2}\right)$. Finally, we obtain from (3.11) and Corollary 1.6.3 in [1] that the operator $\partial F(0)$ satisfies $-\partial F(0) \in \mathcal{H}\left((I-P) H_{\mathcal{B}}^{4}\right.$, $\left.(I-P) L^{2}\right)$.

In order to prove our second assertion, we note that

$$
\partial F(0) Y=-\left[\begin{array}{ll}
A f_{*} & B f_{*} \\
h_{*} & h_{*}
\end{array}\right] \partial_{x}^{4} Y+\left[\begin{array}{ll}
a f_{*} & b f_{*} \\
c h_{*} & c h_{*}
\end{array}\right] \partial_{x}^{2} Y, \quad Y \in(I-P) H_{\mathcal{B}}^{4},
$$

and use the fact that the embedding $H_{\mathcal{B}}^{4} \hookrightarrow L_{2}$ is compact. Since by (a) the derivative $\partial F(0)$ is a sectorial operator, we conclude that the resolvent 
$(\lambda-\partial F(0))^{-1} \in \mathcal{L}\left((I-P) L_{2}\right)$ is compact for all $\lambda$ in a certain right-halfplane. As a direct consequence, the spectrum of $\partial F(0)$ consists only of isolated eigenvalues with finite multiplicity, cf. Theorem III.6.29 in [11]. In order to determine these eigenvalues, we denote by $\phi_{k}:=\sqrt{2 / L} \cos (k \pi x / L), k \geq 1$, the eigenfunctions of the operator

$$
-\partial_{x}^{2}:\left\{f \in H^{2}((0, L), \mathbb{R}): \partial_{x} f=0 \text { for } x=0, L\right\} \rightarrow L_{2}((0, L), \mathbb{R})
$$

corresponding to its (positive) eigenvalues. It is not difficult to see that $Y=$ $(f, h) \in(I-P) H_{\mathcal{B}}^{4}$ if and only if

$$
\left(\sum_{k=1}^{n}\left(f \mid \phi_{k}\right)_{L_{2}} \phi_{k}, \sum_{k=1}^{n}\left(h \mid \phi_{k}\right)_{L_{2}} \phi_{k}\right) \rightarrow_{n \rightarrow \infty}(f, h) \quad \text { in } H^{4} .
$$

Whence, if $\lambda$ is an eigenvalue of $\partial F(0)$, there exists a pair $Y=(f, h) \in$ $(I-P) H_{\mathcal{B}}^{4}$ such that

$$
\begin{gathered}
-A f_{*} \partial_{x}^{4} f-B f_{*} \partial_{x}^{4} h+a f_{*} \partial_{x}^{2} f+b f_{*} \partial_{x}^{2} h=\lambda f, \\
-h_{*} \partial_{x}^{4} f-h_{*} \partial_{x}^{4} h+c h_{*} \partial_{x}^{2} f+c h_{*} \partial_{x}^{2} h=\lambda h .
\end{gathered}
$$

Since $\left\{\phi_{k}: k \geq 1\right\}$ is an orthonormal basis in $(I-P) L_{2}$, relations (3.12)-(3.14) imply that $\lambda$ is an eigenvalue of $\partial F(0)$ if and only if

$$
\left|\begin{array}{ll}
A f_{*}(k \pi / L)^{4}+a f_{*}(k \pi / L)^{2}+\lambda & B f_{*}(k \pi / L)^{4}+b f_{*}(k \pi / L)^{2} \\
h_{*}(k \pi / L)^{4}+c h_{*}(k \pi / L)^{2} & h_{*}(k \pi / L)^{4}+c h_{*}(k \pi / L)^{2}+\lambda
\end{array}\right|=0
$$

for some $k \geq 1$. By computing the solutions of these equations, we obtain the desired assertion.

Proof of Theorem 3.3. Let us first observe that both sequences $\lambda_{1 / 2}^{k} \rightarrow-\infty$, meaning that the stability properties of the trivial solution $Y=0$ of (3.9) are determined by the sign of the eigenvalues with small $k$.

Assume first that (3.2) is satisfied. Then, we infer from (3.10) that spectrum of $\partial F(0)$ is contained in a left-halfplane $\left[\operatorname{Re} \lambda \leq-\omega_{0}\right]$ with $\omega_{0}>0$. From (2.4) and Theorem 9.1.2 in [12] we conclude that the solution $Y=0$ of (3.9) is exponentially stable. Recalling (3.7), we obtain the desired assertion in the case when (3.2) is satisfied.

If, on the other hand, (3.5) is satisfied, then $\lambda_{2}^{1}$ is a positive eigenvalue, and in this case the solution $Y=0$ of (3.9) is unstable, cf. Theorem 9.1.3 in [12]. This completes the proof.

Non-flat equilibria. We notice that Corollary 3.2 does not exclude the existence of non-flat stationary solutions of (1.1) when the less dense fluid lies beneath. By fixing the volume of fluids, that is fixing a flat steady-state $X_{*}=$ $\left(f_{*}, h_{*}\right)$ of $(1.1)$, finding the non-flat solutions of (1.1) under this volume constraint is equivalent to determining the stationary solutions of (3.9). Of course, due to Theorem 3.3, we may aspect non-flat steady-state solutions to exist only if (3.2) is not satisfied. Indeed, it follows from (3.9) and (1.1a) that 
$Y_{\circ}=\left(f_{\circ}, h_{\circ}\right)$ is a stationary solution of $(3.9)$ if and only if $\left(f_{\circ}, h_{\circ}\right)$ solves the following system of linear ordinary differential equations

$$
\left\{\begin{array}{l}
A f_{\circ}^{\prime \prime}+B h_{\circ}^{\prime \prime}-a f_{\circ}-b h_{\circ}=0, \\
f_{\circ}^{\prime \prime}+h_{\circ}^{\prime \prime}-c f_{\circ}-c h_{\circ}=0 .
\end{array}\right.
$$

Particularly, $\alpha Y_{\circ} \in(I-P) H_{\mathcal{B}}^{4}$ is again a stationary solution of (3.9), provided $\alpha \in \mathbb{R}$ is sufficiently small. We infer then from Theorem 3.3 that, if $(3.2)$ is satisfied, this solution converges exponentially fast to the trivial solution $Y=(0,0)$ of $(3.9)$, which is a contradiction.

Let us now determine the solutions of $(3.15)$ which lie in $(I-P) H_{\mathcal{B}}^{4}$. If we multiply the second equation of (3.15) by $-B$ and sum it to the first one we obtain, from $c B=b$, that

$$
(A-B) f_{\circ}^{\prime \prime}+(b-a) f_{\circ}=0 .
$$

The nontrivial solutions of this equation are in $(I-P) H_{\mathcal{B}}^{4}$ if and only if there exists an integer $m \geq 1$, such that

$$
\frac{b-a}{A-B}=\left(\frac{m \pi}{L}\right)^{2}
$$

We assume further on that such an integer $m$ exists. Then, the solutions of (3.15) within $(I-P) H_{\mathcal{B}}^{4}$ form a one-dimensional subspace

$$
N_{m}:=\left\{\alpha Y_{\circ}:=\alpha\left(f_{\circ}, h_{\circ}\right)=\alpha\left(\phi_{m},-\phi_{m}\right): \alpha \in \mathbb{R}\right\}
$$

and, as long as $\alpha Y_{\circ} \in \mathcal{O}_{Y}$, the function $X_{\circ}:=X_{*}+\alpha Y_{\circ} \in \mathcal{O}$ is a stationary solution of (1.1). We notice that the upper boundary $\tilde{f}_{\circ}:=\left(f_{*}+\alpha f_{\circ}\right)+\left(h_{*}+\right.$ $\left.\alpha h_{\circ}\right)=f_{*}+h_{*}$ is flat. This is exactly the situation which is encountered when considering the full problem, cf. [7]. However, in contrast to [7], where the nontrivial steady-state solutions have been obtained in the same regime (3.5) but via bifurcation, in the setting considered herein they are solutions of a linear system of ordinary differential equations with constant coefficients and form linear one-dimensional invariant subspaces of the phase space, i.e. linear center manifolds. The stability properties of those non-flat steady-state solutions are studied below. The case when $m=1$ is special and, in order to analyze it, we introduce the Nikolskii spaces $N_{2, \mathcal{B}}^{s}:=B_{2, \infty, \mathcal{B}}^{s}\left((0, L), \mathbb{R}^{2}\right)$ for $s>0$, cf. [21]. Here, $\mathcal{B}$ denotes the same normal system of boundary operators as in Sect. 2, that is $\mathcal{B}:=\left\{\left.\partial_{x}^{2 l+1}\right|_{x=0, L}: l=0,1\right\}$.

Theorem 3.6. Assume that (3.16) is satisfied for some integer $m \geq 1$. We then have:

(i) If $m \geq 2$, there exists $\alpha_{0}>0$, such that $\alpha Y_{\circ}$ is an unstable steady-state solution of (3.9) for all $|\alpha|<\alpha_{0}$, i.e. $N_{m}^{0}:=\left\{\alpha Y_{\circ}:|\alpha|<\alpha_{0}\right\}$ is a center unstable manifold;

(ii) If $m=1$ we pick $\theta \in(0,1 / 4)$. Then, there exist positive constants $\delta, M$ and $\omega>0$ such that for all $Y_{0} \in(I-P) N_{2, \mathcal{B}}^{4(1+\theta)}$ with $\left\|Y_{0}\right\|_{N_{2, \mathcal{B}}^{4(1+\theta)}} \leq \delta$ the solution of (3.9) exists globally and there exists $\alpha_{*} \in \mathbb{R}$ such that

$$
\left\|Y(t)-\alpha_{*} Y_{\circ}\right\|_{N_{2, \mathcal{B}}^{4(1+\theta)}} \leq M e^{-\omega t}\left\|Y_{0}\right\|_{N_{2, \mathcal{B}}^{4(1+\theta)}}, \quad \forall t \geq 0 .
$$


In this case $N_{1}$ is a center stable manifold.

Remark 3.7. As mentioned in the introduction, the subspaces $N_{m}$ are the first order approximation of the bifurcation branches consisting of steady-state solutions of the Muskat problem studied in [7]. While in [7] all small non-flat equilibria are unstable, here, in the case $m=1$, they build a stable center manifold.

The remainder of this section is devoted to the proof of Theorem 3.6. We start with the assertion (i). If $m \geq 2$, then, as we noticed in the proof of Theorem 3.3, $\lambda_{2}^{1}$ is a positive eigenvalue of the Fréchet derivative $\partial F(0)$. By continuity, we obtain from [11, Theorem IV.3.16], that the unstable spectrum of $\partial F\left(\alpha Y_{\circ}\right)$ consists of a finite number of eigenvalues for small $\alpha$. Whence, the assumptions of Theorem 9.1.3 in [12] are satisfied, and our assertion follows now from this theorem.

In order to prove our second assertion we let $m=1$ and note from $F\left(\alpha Y_{\circ}\right)=0$ for $\alpha Y_{\circ} \in \mathcal{O}_{Y}$, that

$$
\partial F\left(\alpha Y_{\circ}\right)\left[Y_{\circ}\right]=0
$$

which implies, for small $\alpha$, that the spectrum of $\partial F\left(\alpha Y_{\circ}\right)$ consists only of negative eigenvalues excepting the eigenvalue $\lambda=0$. Hence, this case is more subtle, and we need to restrict the problem (3.9) to the context of the Nikolskii spaces $N_{2, \mathcal{B}}^{s}$ to be able to analyze it. Let thus $\theta \in(0,1 / 4)$, and define

$$
\begin{aligned}
& E_{0}:=D_{\partial F(0)}(\theta, \infty):=\left((I-P) H_{\mathcal{B}}^{4},(I-P) L_{2}\right)_{\theta, \infty}=(I-P) N_{2, \mathcal{B}}^{4 \theta}, \\
& E_{1}:=(I-P) N_{2, \mathcal{B}}^{4(1+\theta)}
\end{aligned}
$$

with $P$ the projection defined (3.6), cf. Theorem 4.3.3 in [21]. By standard interpolation arguments, the realisation $\partial F(0): E_{1} \rightarrow E_{0}$ is again an analytic generator, that is $-\partial F(0) \in \mathcal{H}\left(E_{1}, E_{0}\right)$. Furthermore, we have that $F: \mathcal{O}_{\theta} \subset$ $E_{1} \rightarrow E_{0}, \mathcal{O}_{\theta}$ being an open small zero-neighbourhood in $E_{1}$, is a well-defined smooth mapping. This is a consequence of the fact that

$$
N_{2}^{r}((0, L), \mathbb{R}) \times N_{2}^{t}((0, L), \mathbb{R}) \rightarrow N_{2}^{t}((0, L), \mathbb{R}), \quad(f, h) \mapsto f h,
$$

is a continuous bilinear map if $t>0$ and $r>t+1 / 2$, cf. Theorems 3.3 .1 and 3.3.2 in [22]. This setting corresponds to the abstract situation considered in Section 9.2 of [12] where it is shown that there exists a center manifold which attracts at an exponential rate solutions corresponding to small data.

We will identify this, a priori non-unique center manifold (cf. Example 13.7 in [23]), with a zero neighbourhood of the subspace $N_{1}$, and prove in this way our claim Theorem 3.6 (ii). To begin, we let $Q$ be the projection on the subspace $N_{1}$, that is

$$
Q(f, h)=\frac{\left(f \mid \phi_{k}\right)_{L_{2}}-\left(h \mid \phi_{k}\right)_{L_{2}}}{2} Y_{\circ}, \quad(f, h) \in E_{1} .
$$

It is convenient to write (3.9) in the following form

$$
\partial_{t} Y=\mathbb{A} Y+G(Y), \quad t>0, \quad Y(0):=Y_{0},
$$


where $\mathbb{A}:=\partial F(0)$ and $G(Y):=F(Y)-\mathbb{A} Y$. If $r>0$ and $\eta: N_{1} \rightarrow[0,1]$ is a smooth cutoff function such that $\eta(x)=1$ for $\|x\|_{E_{0}}<1 / 2$ and $\eta(x)=0$ for $\|x\|_{E_{0}} \geq 1$, then for small solutions, problem (3.19) is equivalent to the system

$$
\left\{\begin{array}{l}
\partial_{t} x=Q G(\eta(x / r) x+y), \\
\partial_{t} y=(I-Q) \mathbb{A} y+(I-Q) G(\eta(x / r) x+y), \quad t>0
\end{array}\right.
$$

with initial conditions

$$
x(0)=x_{0} \in Q E_{1}=N_{1}, \quad y(0)=y_{0} \in(I-Q) E_{1} .
$$

We recall now Theorem 9.2.2 in [12] (see also Proposition 6.2 in [8]).

Theorem 3.8. (Existence of center manifolds) Given $k \in \mathbb{N}, k \geq 1$, there exists $r_{k} \in(0,1)$ and for each $r \in\left(0, r_{k}\right]$ there is a unique mapping

$$
\sigma \in B C^{k}\left(N_{1},(I-Q) E_{1}\right)
$$

which satisfies

$$
\sigma(0)=0 \quad \partial \sigma(0)=0 .
$$

Moreover

$$
\|\sigma(x)-\sigma(\bar{x})\|_{E_{1}} \leq b\|x-\bar{x}\|_{E_{0}}
$$

for a suitable constant $b$ and $\|\sigma(x)\|_{E_{1}} \leq r$ for all $x \in N_{1}$.

Let $\mathcal{M}:=\left\{(x, \sigma(x)): x \in N_{1}\right\} \subset E_{1}$. Then, $\mathcal{M}$ is a globally invariant one-dimensional $C^{k}$-manifold for the problem (3.19), that is, given $\left(x_{0}, y_{0}\right) \in$ $\mathcal{M}$, the solution $(x, y)$ to (3.19) exists in the large and $(x(t), y(t)) \in \mathcal{M}$ for all $t \geq 0$.

Denote by $z(\cdot)=z(\cdot ; x, \sigma)$ the global solution of the reduced ordinary differential equation

$$
\left\{\begin{array}{l}
z^{\prime}(t)=Q G(\eta(z(t) / r) z(t)+\sigma(z(t))), \quad t \in \mathbb{R} \\
z(0)=x
\end{array}\right.
$$

The function $\sigma$ is the unique fixed point of the following equation

$$
\sigma(x)=\int_{-\infty}^{0} e^{-t(I-Q) \mathbb{A}}(I-Q) G(\eta(z(t) / r) z(t)+\sigma(z(t))) d t,
$$

and, for $\left(x_{0}, y_{0}\right) \in \mathcal{M}$, we have that $(x, y)=\left(z\left(\cdot ; x_{0}, \sigma\right), \sigma\left(z\left(; x_{0}, \sigma\right)\right)\right)$ is the globally defined solution to (3.19).

Let us observe that the mapping $\sigma$ obtained in Theorem 3.8 is $\sigma \equiv 0$. Indeed, this results from the uniqueness of $\sigma$, relation (3.18), and the fact that $F\left(\alpha Y_{0}\right)=0$ for small $\alpha$, Therefore, we have $\mathcal{M}=N_{1}$. We infer then from Proposition 9.2.4 in [12] that solutions of (3.9) which are initial small are attracted at an exponential rate by some element of this center manifold. This concludes the proof of Theorem 3.6 (ii). 


\section{References}

[1] Amann, H.: Linear and Quasilinear Parabolic Problems, vol. I. Birkhäuser, Basel (1995)

[2] Amann, H.: Nonhomogeneous linear and quasilinear elliptic and parabolic boundary value problems. In: Schmeisser, H., Triebel, H. (eds.) Function Spaces, Differential Operators and Nonlinear Analysis. Teubner-Texte zur Math., 133, pp 9-126. Teubner, Stuttgart (1993)

[3] Bertozzi, A.L., Pugh, M.: The lubrication approximation for thin viscous films: the moving contact line with a porous media cut-off of van der Waals interactions. Nonlinearity 7, 1535-1564 (1994)

[4] Escher, J., Laurençot, Ph., Matioc, B.-V.: Existence and stability of weak solutions for a degenerate parabolic system modelling two-phase flows in porous media. Ann. Inst. H. Poincaré Anal. Non Linéaire 28(4), 583-598 (2011)

[5] Escher, J., Hillairet, M., Laurençot, P., Walker, C.: Thin film equations with soluble surfactant and gravity: modeling and stability of steady states. Math. Nachr. 285(2-3), 210-222 (2012)

[6] Escher, J., Matioc, A.-V., Matioc, B.-V.: Modelling and analysis of the Muskat problem for thin fluid layers. J. Math. Fluid Mech. doi:10.1007/ s00021-011-0053-2

[7] Escher, J., Matioc, A.-V., Matioc, B.-V.: A generalised Rayleigh-Taylor condition for the Muskat problem. Nonlinearity 25, 73-92 (2012)

[8] Escher, J., Simonett, G.: A center manifold analysis for the Mullins-Sekerka model. J. Differ. Equ. 143, 267-292 (1998)

[9] Günther, M., Prokert, G.: A justification for the thin film approximation of Stokes flow with surface tension. J. Differ. Equ. 245, 2802-2845 (2008)

[10] Giacomelli, L.: A fourth-order degenerate parabolic equation describing thin viscous flows over an inclined plane. Appl. Math. Lett. 12(8), 107-111 (1999)

[11] Kato, T.: Perturbation Theory for Linear Operators. Springer, Berlin (1995)

[12] Lunardi, A.: Analytic Semigroups and Optimal Regularity in Parabolic Problems. Birkhäuser, Basel (1995)

[13] Matioc, B.-V.: Nonnegative global weak solutions for a degenerate parabolic system modeling thin films driven by capillarity. Proc. Roy. Soc. Edinburgh Sect. A (to appear)

[14] Matioc, B.-V., Prokert, G.: Hele-Shaw flow in thin threads: a rigorous limit result. Interfaces Free Bound. (to appear)

[15] Novick-Cohen, A., Shishkov, A.: The thin film equation with backward forcing. Preprint 
[16] Oron, A., Davis, S.H., Bankoff, G.: Long-Scale Evolution of Thin Liquid Films. Rev. Mod. Phys. 69(3), 931-980 (1997)

[17] Dal Passo, R., Giacomelli, L., Shishkov, A.: The thin film equation with nonlinear diffusion. Commun. Partial Differ. Equ. 26(9\&10), 1509-1557 (2001)

[18] Pazy, A.: Semigroups of Linear Operators and Applications to partial Differential Equations. Springer, New York (1983)

[19] Prokert, G.: Parabolic evolution equations for quasistationary free boundary problems in capillary fluid mechanics. Dissertation, Technische Universiteit Eindhoven (1997)

[20] Taranets, R.M., Shishkov, A.E.: Singular Cauchy problem for the equation of flow of thin viscous films with nonlinear convection. Ukrainian Math. J. 58(2), 280-303 (2006)

[21] Triebel, H.: Interpolation Theory, Function Spaces, Differential Operators. Johann Ambrosius Barth Verlag, Heidelberg (1995)

[22] Triebel, H.: Theory of Function Spaces. Birkhäuser, Basel (1983)

[23] Verhulst, F.: Nonlinear Differential Equations and Dynamical Systems. Springer, Berlin (1990)

Joachim Escher and Bogdan-Vasile Matioc

Institut für Angewandte Mathematik

Leibniz Universität Hannover

Welfengarten 1

30167 Hannover

Germany

e-mail: matioc@ifam.uni-hannover.de

Joachim Escher

e-mail: escher@ifam.uni-hannover.de

Received: 7 September 2011.

Accepted: 23 March 2012. 